\title{
Evaluación de la suplementación de creatina sobre el desempeño productivo y variables bioquímicas en lechones al destete
}

\author{
Cayo, P.'; More, M. ${ }^{2}$; Aragón, E. ; Mena, R. ${ }^{1}$; Quisirumbay, J.G. ${ }^{1,3}$ \\ ${ }^{1}$ Facult. Med. Vet. y Zoot. Univ. Central, Ecuador. ${ }^{2}$ Esc. Prof. Ing. Agropec. Facult. Cs. Agrar. \\ Univ. Nac. San Antonio, Cusco, Perú. ${ }^{3}$ Centro Exp. Fac. Med. Vet. \& Zoot, Univ. Central Ecuador. \\ E-mail: jrquisirumbay@uce.edu.ec
}

\begin{abstract}
Resumen
Cayo, P.; More, M.; Aragón, E.; Mena, R.; Quisirumbay, JG.: Evaluación de la suplementación de creatina sobre el desempeño productivo y variables bioquímicas en lechones al destete. Rev. Vet. 32: 2, 230-233, 2021. El destete es considerado como el período más estresante en la producción porcina y se asocia con trastornos intestinales, musculares y bajo rendimiento productivo. Estudios demuestran que la suplementación de creatina favorece la síntesis proteica. El objetivo de este trabajo de investigación fue evaluar el efecto de la suplementación de creatina en lechones mediante la medición del rendimiento productivo y pruebas bioquímicas en sangre. El trabajo de investigación se realizó en el Centro Experimental Uyumbicho (CEU) perteneciente a la Facultad de Medicina Veterinaria y Zootecnia de la Universidad Central del Ecuador. Se utilizó un total de 16 lechones (Landrace x Yorkshire $\mathrm{x}$ Pietrain $\mathrm{x}$ Duroc). Los lechones se distribuyeron de manera aleatoria en cada uno de los 4 tratamientos: $\mathrm{T} 1=$ testigo 1 (sin suplemento lácteo), $\mathrm{T} 2=$ testigo 2 (suplemento lácteo), $\mathrm{T} 3=$ suplemento lácteo + creatina $(0.5 \%), \mathrm{T} 4=$ suplemento lácteo + creatina $(1 \%)$. Los lechones que recibieron creatina al $1 \%$ presentaron el mayor consumo diario de alimento $(0.432 \mathrm{~kg} /$ día) y la mayor ganancia diaria de peso $(0.336 \mathrm{~kg} /$ día) (42 días edad) y la mejor conversión alimenticia $(1.288 \mathrm{~kg} / \mathrm{kg}$ ) (62 días de edad). La enzima creatina cinasa presentó el valor más elevado (2007.5 UL) en los lechones del grupo testigo 1. El uso de un sustituto lácteo y la suplementación de creatina mitiga los efectos adversos post-destete en lechones.
\end{abstract}

Palabras clave: lechones, destete, creatina, suplemento

\begin{abstract}
Cayo, P.; More, M.; Aragón, E.; Mena, R.; Quisirumbay, G.J.: Evaluation of creatine supplementation on productive performance and biochemical variables in piglets at weaning. Rev. Vet. 32: 2, 230-233, 2021. Weaning is considered the most stressful period in pig production and is associated with intestinal and muscular disorders and poor productive performance. Studies show that creatine supplementation favors protein synthesis. The objective of this research was to evaluate the effect of creatine supplementation in piglets by measuring productive performance and biochemical tests in blood. The research was carried out at the Uyumbicho Experimental Center (UEC) belonging to the Faculty of Veterinary Medicine and Zootechnics of the Central University of Ecuador. A total of 16 piglets (Landrace $\mathrm{x}$ Yorkshire $\mathrm{x}$ Pietrain $\mathrm{x}$ Duroc) were used. The piglets were randomly distributed in each of the 4 treatments: $\mathrm{T} 1=$ control 1 (without milk supplement), $\mathrm{T} 2=$ control 2 (milk supplement), $\mathrm{T} 3=$ milk supplement + creatine $(0.5 \%), \mathrm{T} 4=$ milk supplement + creatine $(1 \%)$. Piglets that received $1 \%$ creatine had the highest daily feed intake $(0.432 \mathrm{~kg} / \mathrm{day})$ and the highest daily weight gain $(0.336 \mathrm{~kg} /$ day $)$ ( 42 days old) and the best feed conversion rate $(1.288$ $\mathrm{kg} / \mathrm{kg}$ ) (62 days old). The creatine kinase enzyme presented the highest value (2007.5 UL) in the piglets of control group 1. The use of a milk substitute and creatine supplementation mitigates the adverse effects post-weaning in piglets.
\end{abstract}

Key words: piglets, weaning, creatine, supplement.

\section{INTRODUCCIÓN}

El destete, estrés por transporte y estrés térmico, tienen el potencial de incrementar la incidencia de enfermedades en lechones, especialmente cuando estos ocurren simultáneamente ${ }^{25}$. Durante el destete, el es- trés puede ser inducido por la separación del lechón de la madre, reubicación y mezcla de camadas ${ }^{3,26}$.

La primera semana después del destete es considerado como el período más estresante en lechones y se asocia con trastornos intestinales y presentación de diarreas ${ }^{23}$. Se evidencia deterioro del desarrollo de las 
funciones de barrera del tracto gastro-intestinal, que conduce a consecuencias perjudiciales permanentes para la salud intestinal durante la vida del cerdo ${ }^{14,16}$.

El destete provoca una reducción abrupta en el consumo de alimento generando disminución de los procesos digestivos, destrucción de la morfología intestinal ${ }^{11}$ con pérdida de la actividad enzimática del borde en cepillo y menor digestibilidad de los nutrientes ${ }^{5,9}$. La proteína muscular es utilizada como fuente de energía, deteriorándose el desarrollo muscular del lechón, lo cual se refleja finalmente en un estado de salud inadecuado y un menor rendimiento productivo ${ }^{16}$.

Para combatir los efectos negativos del destete sobre la salud del lechón se han implementado varias estrategias entre las cuales se encuentra: manejo de la edad de destete, acondicionamiento ambiental, manipulación nutricional y uso de aditivos a través de la dieta $^{7,10,20}$. La creatina participa en la regeneración rápida y anaeróbica de ATP en los músculos esqueléticos a través del fosfato de creatina. El fosfato de creatina dona su fosfato de alta energía al ATP ${ }^{24}$.

La evaluación en lechones sugiere que las necesidades absolutas de creatina en neonatos en crecimiento son mayores que las suministradas por la leche porcina (12.7 vs $2.8 \mathrm{mmol}$ por semana, respectivamente); como tal, los recién nacidos dependen de la síntesis de creatina de novo. El $75 \%$ de las necesidades de creatina deben provenir de síntesis endógena de arginina y metionina ${ }^{2}$.

El período neonatal de los lechones se caracteriza por un rápido desarrollo y tasas muy altas de síntesis de proteínas para apoyar el crecimiento. La mayor demanda de arginina para apuntalar la síntesis de creatina, puede limitar la disponibilidad de arginina para la síntesis de proteínas. Estudios demuestran que la suplementación de creatina favorece la síntesis proteica en hígado y riñón ${ }^{6}$.

Tabla 1. Contenido nutricional, dieta comercial utilizada.

\begin{tabular}{lcc}
\hline & fase 2 & fase 3 \\
\hline edad (días) & $28-49$ & $50-70$ \\
proteína cruda (min.) (\%) & 21 & 18 \\
grasa (min.) (\%) & 5 & 4 \\
fibra cruda (máx.) (\%) & 5 & 5 \\
cenizas (máx.) (\%) & 7 & 7 \\
humedad (máx.) (\%) & 12 & 12 \\
\hline
\end{tabular}

La Fase 1 (1-27 días de edad) del programa de alimentación la recibieron todos los lechones antes del destete.
Las células miogénicas en cultivo, expuestas al estrés oxidativo, han disminuido la proliferación y diferenciación, y la creatina atenuó estos efectos ${ }^{17}$. $\mathrm{La}$ creatina también regula al alza la expresión de una serie de factores tróficos en el músculo, incluido el IGF-1 (factor de crecimiento insulínico tipo 1), que puede estimular la síntesis de proteínas a través de la activación de los intermedios de la vía mTOR ${ }^{18}$

El destete en lechones disminuye la síntesis proteica, crecimiento muscular y rendimiento productivo. Por lo tanto, el objetivo de este trabajo de investigación fue evaluar el efecto de la suplementación de creatina en lechones mediante la medición del rendimiento productivo y pruebas bioquímicas en sangre.

\section{MATERIAL Y MÉTODOS}

El presente trabajo de investigación se realizó en el módulo porcino del Centro Experimental Uyumbicho (CEU) perteneciente a la Facultad de Medicina Veterinaria y Zootecnia de la Universidad Central del Ecuador. Se utilizó un diseño completamente al azar, cada tratamiento tuvo 2 repeticiones y cada repetición incluyó 2 lechones. Utilizándose un total de 16 lechones, la línea genética utilizada fue Landrace $\mathrm{x}$ Yorkshire $\mathrm{x}$ Pietrain $x$ Duroc.

Los lechones fueron destetados a los 28 días de edad y se distribuyeron de manera aleatoria en cada uno de los 4 tratamientos: T1 = testigo 1 (sin suplemento lácteo), $\mathrm{T} 2=$ testigo 2 (suplemento lácteo), T3 = suplemento lácteo + creatina $(0.5 \%)$, y T4 = suplemento lácteo + creatina (1\%). El suplemento lácteo con o sin creatina fue administrado por un periodo de 15 días (28 hasta los 42 días de edad). Los lechones fueron alimentados con 2 fases de un alimento comercial cuya composición se muestra en la Tabla 1.

El rendimiento productivo (ganancia diaria de peso, consumo de alimento y conversión alimenticia) se midió a los 42 y 62 días de edad. Las pruebas bioquímicas en sangre (creatina cinasa (CK), urea, lactato deshidrogenasa (LDH), creatinina, proteínas totales, albúminas y globulinas) se midieron a los 42 días. El análisis estadístico se realizó mediante proc GLM en el sistema SAS versión 9.0 .

\section{RESULTADOS}

La Tabla 2 muestra que a los 42 días de edad los lechones que recibieron creatina al $1 \%$ presentaron el mayor consumo diario de alimento $(0.432 \mathrm{~kg} / \mathrm{d}$ ía) y la mayor ganancia diaria de peso $(0.336 \mathrm{~kg} /$ día $)$ al com-

Tabla 2. Rendimiento productivo en lechones a los 42 días de edad.

\begin{tabular}{|c|c|c|c|c|c|}
\hline variable & $\begin{array}{c}\text { testigo } \\
1\end{array}$ & $\begin{array}{c}\text { testigo } \\
2\end{array}$ & $\begin{array}{c}\text { creatina } \\
0.5 \%\end{array}$ & $\begin{array}{c}\text { creatina } \\
1 \%\end{array}$ & p-valor \\
\hline consumo diario alimento $(\mathrm{kg} / \mathrm{dí} a)$ & 0.395 & 0.383 & 0.377 & 0.432 & 0.374 \\
\hline ganancia diaria de peso (kg/día) & 0.256 & 0.296 & 0.290 & 0.336 & 0.797 \\
\hline conversión alimenticia $(\mathrm{kg} / \mathrm{kg})$ & 1.677 & 1.301 & 1.317 & 1.333 & 0.753 \\
\hline
\end{tabular}


Tabla 3. Rendimiento productivo en lechones a los 62 días de edad.

\begin{tabular}{lccccc}
\hline \multirow{2}{*}{ variable } & testigo & testigo & creatina & creatina & p-valor \\
& 1 & 2 & $0.5 \%$ & $1 \%$ & 0.717 \\
consumo diario alimento $(\mathrm{kg} / \mathrm{dí}$ ) & 0.703 & 0.690 & 0.697 & 0.571 \\
ganancia diaria de peso $(\mathrm{kg} / \mathrm{dia})$ & 0.617 & 0.614 & 0.618 & 0.660 & 0.758 \\
conversión alimenticia $(\mathrm{kg} / \mathrm{kg})$ & 1.388 & 1.319 & 1.432 & 1.288 & 0.736 \\
\hline
\end{tabular}

Tabla 4. Variables bioquímicas evaluadas en lechones a los 42 días de edad.

\begin{tabular}{lccccc}
\hline variable & testigo & testigo & creatina & creatina & p-valor \\
\hline $\mathrm{CK}(\mathrm{UL})$ & 1 & 2 & $0.5 \%$ & $1 \%$ & 0.78 \\
urea $(\mathrm{mMol} / \mathrm{L})$ & 2007.50 & 1377.50 & 1604.00 & 1450.00 & 0.66 \\
$\mathrm{LDH}(\mathrm{UL})$ & 2.95 & 3.20 & 1.90 & 2.90 & 0.65 \\
creatinina $(\mathrm{uMol} / \mathrm{L})$ & 906.00 & 793.00 & 851.50 & 1088.50 & 0.42 \\
proteínas totales $(\mathrm{g} / \mathrm{dl})$ & 111.55 & 129.00 & 123.40 & 113.15 & 0.60 \\
albúmina $(\mathrm{g} / \mathrm{dl})$ & 6.00 & 6.20 & 6.50 & 6.20 & 0.84 \\
globulina $(\mathrm{g} / \mathrm{dl})$ & 2.99 & 2.89 & 3.04 & 3.18 & 0.59 \\
\hline
\end{tabular}

pararlo frente a los demás grupos. Sin embargo, los lechones del grupo testigo 2 fueron los más eficientes pues tuvieron la conversión alimenticia más baja (1.301 $\mathrm{kg} / \mathrm{kg}$ ).

En la Tabla 3 se evidencia que los mejores resultados en el rendimiento productivo de lechones a los 62 días edad lo presentaron aquellos que recibieron creatina al $1 \%$. Los valores obtenidos fueron 0.717 (kg/día), $0.660(\mathrm{~kg} / \mathrm{d}$ ía) y $1.288(\mathrm{~kg} / \mathrm{kg})$ para consumo diario de alimento, ganancia diaria de alimento y conversión alimenticia respectivamente. Los lechones del grupo creatina $(0.5 \%)$ fueron los menos eficientes a los 62 días de edad ya que presentaron una conversión alimenticia de $1.432(\mathrm{~kg} / \mathrm{kg})$.

Respecto a las variables bioquímicas analizadas, como se muestra en la tabla 4, se encontró que la enzima CK presentó el valor más elevado (2007.5 UL) en los lechones del grupo testigo 1, es decir de aquellos que no recibieron ningún tipo de suplementación. Si bien la diferencia no fue significativa, la tendencia fue superior en estos lechones frente al resto de tratamientos.

La enzima LDH tuvo el valor más elevado en el grupo que recibió creatina al 1\% (1088.5 UL). Los valores de las demás variables analizadas (urea, creatinina, proteínas totales, albúmina y globulina) no presentaron diferencia entre los tratamientos.

\section{DISCUSIÓN}

Posterior al nacimiento, el tracto gastrointestinal de los lechones pasa por un proceso de maduración dinámica, que incluye aspectos morfológicos y funcionales como los relacionados al sistema inmune asociado a la mucosa intestinal ${ }^{19}$.

Los órganos digestivos están morfo-fisiológicamente adaptados a la alimentación láctea (calostro y leche), alcanzando así un alto desempeño de estos animales durante la fase de lactancia ${ }^{21}$. Sin embargo, en la producción porcina moderna, el destete es uno de los períodos más críticos durante la vida del cerdo debido a la alta exposición a factores estresantes ${ }^{1}$.

Los resultados encontrados en esta investigación muestran que no hubo diferencia significativa en el rendimiento productivo ( 42 y 62 días de edad) entre los diferentes tratamientos utilizados, similar a lo reportado por varios grupos de investigación. En un estudio en el cual suplementaron creatina a lechones (4 días de edad) a un nivel de $0.20 \%$ a través de un sustituto lácteo, no encontraron diferencias significativas en el depósito de creatina en músculo ni en el desempeño productivo ${ }^{13}$.

De manera similar, en otro trabajo, lechones destetados se suplementaron con dos niveles de creatina $(0.10$ y $0.5 \%)$ durante 6 días, y en ninguno de los niveles se encontraron diferencias significativas en la ganancia de peso ni en la eficiencia alimenticia ${ }^{8}$. En dos estudios en cerdos en finalización se utilizó creatina a niveles de $0.55 \%$ y $0.8 \%$ con resultados variables sobre la calidad de carne ${ }^{12,22}$.

Se considera que la suplementación de creatina mejora el vigor de los lechones, así como la disponibilidad de energía para la síntesis proteica y el crecimiento muscular, mitigando de esta manera los efectos adversos del destete.

La suplementación de creatina al 1\% mostró -a los 62 días de edad- la más alta ganancia diaria de peso y la más baja conversión alimenticia, lo cual hace pensar que el efecto de la creatina se observa con el paso del tiempo y podríamos tener diferencias significativas en el rendimiento productivo cuando los cerdos fueran llevados hasta mayores edades.

Al analizar las variables bioquímicas tampoco hubo diferencia significativa entre los tratamientos. Sin embargo, todos los tratamientos que recibieron suplemento lácteo presentaron los valores más bajos de $\mathrm{CK}$, mientras que el grupo testigo (1) que no recibió ningún tipo de suplemento, presentó el valor más alto (2007.5 UL) lo cual es indicio de un desgaste muscular, el cual estaría asociado a la movilización de proteína a causa del estrés que genera el destete. 
Dicho desgaste muscular concuerda con la menor ganancia diaria de peso que presentó el grupo $1(0.256$ g/día) a los 42 días de edad. A pesar de ello, dicho efecto no se mantuvo cuando la medición se realizó a los 62 días de edad. Los niveles más bajos de $\mathrm{CK}$ en el resto de grupos estaría vinculado al consumo del suplemento lácteo como tal, cuyos ingredientes aportan una fuente de aminoácidos de calidad y altamente digestibles ${ }^{15}$.

No se debe descartar el efecto benéfico de la creatina sobre la protección muscular, así por ejemplo, en un estudio realizado en ratas se encontró que la suplementación de creatina durante 21 días previno la proteólisis y la atrofia muscular ${ }^{4}$, con lo cual se espera tener una menor presencia de CK en sangre.

El destete en lechones genera un desgaste muscular y menor rendimiento productivo. El uso de un sustituto lácteo y la suplementación de creatina mitigan los efectos adversos post-destete, sin embargo, debe considerarse un mayor tiempo de evaluación.

\section{REFERENCIAS}

1. Boundry C, Dehoux JP, Portetelle D, Buldgen A. 2008 Bovine colostrum as a natural growth promoter for newly weaned piglets: a review. Biotechnol Agron Soc Envir 12: 2, 157-170.

2. Brosnan JT et al. 2009. Creatine synthesis is a major metabolic process in neonatal piglets and has important implications for amino acid metabolism and methyl balance. $J$ Nutr 139: 7, 1292-1297.

3. Campbell JM, Crenshaw JD, Polo J. 2013. The biological stress of early weaned piglets. J Anim Sci Biotechnol 4: $1,19$.

4. Cella PS et al. 2020. Creatine supplementation in Walker-256 tu mor-bearing rats prevents skeletal muscle atrophy by attenuating systemic inflammation and protein degradation signaling. Eur J Nutr 59: 2, 661-669.

5. Cunningham JG, Klein BG. 2014. Fisiología Veterinaria, 5 ta ed., Elsevier, Barcelona, p. 298-317.

6. Dinesh OC, Bertolo RF, Brunton JA. 2017. Creatine supplementation to total parenteral nutrition improves creatine status and supports greater liver and kidney protein synthesis in neonatal piglets. Pediatr Res 83: 1, 135-141.

7. Gresse $\mathbf{R}$ et al. 2017. Gut microbiota dysbiosis in postweaning piglets: understanding the keys to health. Trends Microbiol 25: 10, 851-873.

8. Guzik AC, Southern LL, Matthews JO, Bidner TD, Ladner JP. 2000. Ornithine alpha-ketoglutarate and creatine effects on growth and plasma metabolites of nursery pigs. J Anim Sci 78: 4, 1022-1028.

9. Kluess J, Schoenhusen U, Souffrant WB, Jones PH, Miller BG. 2010. Impact of diet composition on ileal digestibility and small intestinal morphology in earlyweaned pigs fitted with a T-cannula. Animal 4: 4, 586-594.
10. Lalles JP, Bosi P, Smidt H, Stokes CR. 2007. Nutritional management of gut health in pigs around weaning. Proc Nutr Soc 66: 2, 260-268.

11. Leonard SG, Sweeney T, Bahar B, Lynch BP, O'Doherty JV. 2011. Effects of dietary seaweed extract supplementation in sows and post-weaned pigs on performance, intestinal morphology, intestinal microflora and im mune status. Br J Nutr 106: 5, 688-699.

12. Li JL et al. 2016. Effect of creatine monohydrate supplementation on carcass traits, meat quality and postmortem energy metabolism of finishing pigs. Anim Prod Sci 56: 1 , 48-54.

13. Miller ER et al. 1962. Creatine in diet of the baby pig. $J$ Anim Sci 21: 3, 458-460.

14. Moeser AJ, Pohl CS, Rajput M. 2017. Weaning stress and gastrointestinal barrier development: implications for lifelong gut health in pigs. Anim Nutr 3: 4, 313-321.

15. National Research Council. 2012. Nutrient requirements of swine, $11^{\text {th }}$ ed., National Academies Press, Washington, p. 289-295.

16. Puskle JR, Turpin DL, Kim JC. 2018. Gastrointestinal tract (gut) health in the young pig. Anim Nutr 4: 2, 187-196.

17. Sestili P et al. 2009. Creatine supplementation prevents the inhibition of myogenic differentiation in oxidatively injured $\mathrm{C} 2 \mathrm{C} 12$ murine myoblasts. Mol Nutr Food Res 53: 9, 1187-1204.

18. Sestili $P$ et al. New insights into the trophic and cytoprotective effects of creatine in in vitro and in vivo models of cell maturation. Amino Acids 48: 8, 1897-1911.

19. Simon O. 2010. An interdisciplinary study on the mode of action of probiotics in pigs. J Anim Feed Sci 19: 2, 230-243.

20. Solá OD, Gasa J. 2017. Feeding strategies in pig production: Sows and their piglets. Anim Feed Sci Technol 233: 34-52.

21. Souza TC et al. 2012. Cambios nutrimentales en el lechón y desarrollo morfofisiológico de su aparato digestivo. Vet Mex 43: 2, 155-173

22. Stahl CA, Allee GL, Berg EP. 2001. Creatine monohydrate supplemented in swine finishing diets and fresh pork quality: II. Commercial applications. J Anim Sci 79: 12, 3081-3086.

23. Sugiharto S, Hedemann MS, Lauridsen C. 2014. Plasma metabolomic profiles and immune responses of piglets after weaning and challenge with E. coli. J Anim Sci Biotechnol 5: $1,17$.

24. Vallet JL, Miles JR, Rempel LA. 2013. Effect of creatine supplementation during the last week of gestation on birth intervals, stillbirth, and preweaning mortality in pigs. $J$ Anim Sci 91: 5, 2122-2132.

25. Varley M, Wiseman J. 2001. The weaner pig: nutrition and management, 1st ed., CABI, New York, p. 223-243.

26. Wijtten PJ, Meulen J, Verstegen MW. 2011. Intestinal barrier function and absorption in pigs after weaning: a review. $B r J N$ utr 105: 7, 967-981. 